\title{
Prevalence of Cutaneous Disorders in Patients with Polycystic Ovary Syndrome
}

\author{
Mohammed H. Mostafa $^{1^{*}(0)}$, Nader F. Ragab ${ }^{2}$, Ghada F. Mohammed ${ }^{2}$ \\ ${ }^{1}$ Department of Obstetrics and Gynecology, Faculty of Medicine, Ain Shams University, Cairo, Egypt \\ ${ }^{2}$ Department of Dermatology, Venereology and Andrology, Faculty of medicine, Ain Shams University, Cairo, Egypt \\ Email:*moh_marwa_omar@hotmail.com,nader_ragab@hotmail.com,ghadamom@yahoo.com
}

How to cite this paper: Mostafa, M.H., Ragab, N.F. and Mohammed, G.F. (2020) Prevalence of Cutaneous Disorders in $\mathrm{Pa}$ tients with Polycystic Ovary Syndrome. Open Journal of Obstetrics and Gynecology, 10, 1246-1264.

https://doi.org/10.4236/ojog.2020.1090116

Received: July 7, 2020

Accepted: September 20, 2020

Published: September 23, 2020

Copyright (c) 2020 by author(s) and Scientific Research Publishing Inc. This work is licensed under the Creative Commons Attribution International License (CC BY 4.0).

http://creativecommons.org/licenses/by/4.0/

\begin{abstract}
Purpose: To determine the prevalence of cutaneous disorders of PCOS and indicate the specific cutaneous lesions that have reliable association with PCOS. Methods: 134 female participants had met the criteria to be diagnosed as PCOS. Dermatological examination regarding hirsutism, acne, androgenic alopecia and acanthosis nigricans as well as any other cutaneous manifestations and assessment of each as regards duration, distribution and scoring was done. Hormonal assay for FSH/LH was done together with ultrasound examination. Results: The study included 134 female participants who had met the criteria to be diagnosed as PCOS; the range of age was 18 - 35 years, with a mean $(26.31 \pm 4.47$ years $)$. Among the PCOS patients at least one cutaneous disorder was found in 18 patients (13.4\%), two cutaneous disorders in 44 patients (32.8\%), three cutaneous disorders in 47 patients (35.1\%), four cutaneous disorders in 24 (17.9\%) and five cutaneous disorders were found in only one patient $(0.7 \%)$. Statistically, there was a significant positive correlations between hirsutism, acne, acanthosis nigricans and LH/F.S.H ratio ( $\mathrm{p}<$ $0.001)$, and total testosterone level $(\mathrm{p}=0.019, \mathrm{p}<0.001, \mathrm{p}<0.001)$ respectively. Statistically, there was non-significant difference between patients with and without seborrheic dermatitis regarding LH/F.S.H ratio and total testosterone level $(p>0.5)$. Conclusions: The current study showed the possible association of hirsutism, acne, acanthosis nigricans, and seborrheic dermatitis with PCOS. These cutaneous manifestations could be relied upon as potential cutaneous indicator of PCOS. Using Rotterdam criteria women who met criteria of PCOS had a higher prevalence of hirsutism, acne, AN, androgenic alopecia and seborrheic dermatitis.
\end{abstract}

\section{Keywords}

Hirsutism, Acanthosis Negricans, Acne, Seborrheic Dermatitis 


\section{Introduction}

Polycystic Ovary Syndrome (PCOS) is recognized as the most common endocrine disorder of reproductive-aged women around the world [1]. It affects $2 \%$ to $7 \%$ of women in the general population [2]. The pathogenesis underlying these clinical features is poorly understood. Gonadotropic dysregulation, genetics, and environmental factors have been implicated [3].

The diagnostic criteria for PCOS continue to evolve, but the 2003 Rotterdam consensus criteria remain widely used. These criteria require at least 2 of the following findings for diagnosis; oligoanovulation, polycystic ovaries on transvaginal ultrasonography, and clinical signs or biochemical evidence of hyperandrogenism [4].

Patients with PCOS are frequently first seen by a dermatologist. It is estimated that $72 \%$ to $82 \%$ of women with PCOS are seen with cutaneous signs classically associated with hyperandrogenism such as acne, hirsutism, and androgenic alopecia [5]. Hyperandrogenism may also manifest as acanthosis nigricans [6].

Hirsutism is the most common clinical manifestation of hyperandrogenism in women [7]. Approximately $60 \%$ to $70 \%$ of women with PCOS have hirsutism [8]. Hirsutism is defined as excessive terminal hair growth that takes on a male pattern distribution [9]. The hair type present in most women with a hormonal hyperandrogenic disorder is coarse, thickened, pigmented, and long and is called terminal hair. Typically, the onset of hirsutism in PCOS follows menarche, although a minority of premenarche girls have earlier onset of pubic hair development and some degree of hirsutism. The hirsutism in PCOS is more pronounced. The presence of substantial numbers of terminal hairs over the chin, neck, lower face, and sideburns indicates the presence of androgen excess. Similarly, excessive hair growth on the lower back, sternum, abdomen, shoulders, buttocks, perineal area, and inner thighs is considered abnormal. Several clinical assessment scales have been used in the grading of hirsutism [10].

During adolescence, acne should not be considered a substitute of hyperandrogenism [5], although girls with severe acne or acne that is resistant to oral and topical agents, including isotretinoin, may have a $40 \%$ likelihood of developing PCOS [11]. About one third of women with PCOS, particularly younger women, demonstrate acne [12]. When acne persists after adolescence or is exacerbated in the mid-20s or -30s, hyperandrogenemia is common, and acne may be considered a clinical sign of hyperandrogenism. Those presenting with acne alone may have serum free $\mathrm{T}$ levels as high as those seen in hyperandrogenic disease states, demonstrating hirsutism without acne [13].

Alopecia in females may present as diffuse pattern of thinning hair over the vertex of the scalp with the frontal hair line commonly preserved. It is a poor predictor of biochemical hyperandrogenemia, and low serum iron levels and aging are more common causes of hair loss in women [14]. In the setting of androgen excess, androgen-sensitive hair follicles shorten during the anagen phase, resulting in miniaturization of the scalp hair, less scalp coverage, and alopecia. 
The pattern of hair loss in women with hyperandrogenism is variable. For example, although hair loss patterns in women with hyperandrogenemia typically involve the vertex, crown, or a diffuse pattern, women with more severe hyperandrogenemia may experience bitemporal hair loss and loss of the frontal hairline [15]. While the actual prevalence of alopecia in women with PCOS is relatively low compared with other androgenic symptoms (approximately 5\%), an association with polycystic ovaries has been reported [16].

Acanthosis nigricans is characterized by brown and velvety hyperpigmentation of the skin with accentuation on skin folds. It is most commonly observed in the neck and intertriginous areas, such as armpits, groins and inframammary region. It is reported in 5\% of the patients with PCOS [17]. Excessive binding of serum insulin to IGF-1 receptors in peripheral tissues determines the proliferation of keratinocytes and fibroblasts; hence, acanthosis nigricans is a cutaneous manifestation of hyperinsulinemia, and not just of obesity. Despite being mostly associated with obesity, PCOS and diabetes, acanthosis nigricans may be associated with genetic diseases, drug reaction (nicotinic acid) and malignancies [6].

PCOS shows reproductive and metabolic complications that must be diagnosed and treated early due to the risk of infertility, endometrial cancer and plurimetabolic syndrome. Besides these complications, PCOS is associated with high morbidity due to aesthetic aspects that negatively affect women's self esteem. Knowledge about the physiopathologic mechanisms of this syndrome is very important for an appropriate therapeutic approach [18].

Early detection of PCOS cases could be aided by picking up cutaneous lesions that are specifically associated with PCOS. In addition, many skin manifestations were regarded clinically suspicious as to diagnose PCOS such as acne, hirsutism, androgenic alopecia, acanthosis nigricans and seborrheic dermatitis. However some of these skin lesions are present in female patients who have not been diagnosed as having PCOS. The aim of the current study was to determine the prevalence of cutaneous disorders of PCOS and indicate the specific cutaneous lesions that have reliable association with PCOS. These could be used as a specific marker for early diagnosis of highly suspect patients with PCOS.

\section{Materials and Methods}

The present study included (134) female patients that were diagnosed as having polycystic ovary syndrome. They were selected from patients attending dermatology and infertility outpatient clinics at Ain Shams University hospitals over a period of 9 months between May 2016 and February 2017.

An informed verbal consent was obtained for study participation and specimen collection according to Ain Shams University Hospitals ethics committee.

Inclusion criteria:

- Patients at childbearing period (18 to 35 years).

- Participants were diagnosed of polycystic ovary syndrome (PCOS) according to Rotterdam criteria (Rotterdam consensus workshop, 2004) where at least 
two of the following criteria should be present to establish the diagnosis of polycystic ovaries.

a) Oligoovulation and/or anovulation (no symptoms of ovulation).

b) Excess androgen activity:

- Clinical hyperandrogenism is the second defining characteristic of polycystic ovary syndrome (PCOS). This is manifested clinically by hirsutism, acne, and male pattern balding. In rare instances, increased muscle mass, deepening of the voice, or clitoromegaly may occur [19].

- Biochemically: In women who present with hyperandrogenic symptoms, we measure serum total testosterone concentrations because it provides the best overall estimate of androgen production in hirsute women [20]. The criteria of biochemical hyperandrogenemia were total testosterone $(\mathrm{T})>0.68 \mathrm{ng} / \mathrm{mL}$ [21].

a) Polycystic ovaries (by gynecologic ultrasound) the presence of more than 12 follicles with diameter $2-9 \mathrm{~mm}$ arranged peripherally or scattered around a dense stroma.

Exclusion Criteria:

- Other endocrinopathies, such as congenital adrenal hyperplasia (21-hydroxylase deficiency), Cushing's syndrome, hyperprolactinemia, androgen-secreting neoplasms, and other pituitary or adrenal disorders.

- Major medical disorders and/or use of hormonal and/or metabolic drugs within the previous 2 months.

- Women presented with ovarian pathology (cysts, tumors, etc.).

\subsection{Methodology}

Patients were included sequentially in the study provided that they met the inclusion criteria and lacked the exclusion criteria. They were screened for the presence of skin lesions.

The following data were collected from all patients:

Personal history: name, age, occupation, address, marital status, duration of marriage, number of children, duration of infertility, any special habits.

Menstrual history: Regularity (regular if every 28 days \pm 7 days), amount (how many pads (soaked, 1/2 soaked, spotting), duration (average if $2-6$ days) and symptoms of ovulation (regular menses, spasmodic dysmenorrhea, premenstrual tension, midcyclic triad discharge, mid cyclic pain, spotting) [22].

Other symptoms suggestive of PCOS: Infertility (1 ry or 2 ry, period of infertility, investigations done, treatment received) and obesity.

Obstetric history: Gravidity, parity and abortions.

Medical history: Including psychiatric problems, diabetes, and hypertension.

Drug history.

\subsection{Examination}

Dermatological examination: 
Dermatological examination regarding hirsutism, acne, androgenic alopecia and acanthosis nigricans as well as any other cutaneous manifestations and assessment of each as regards duration, distribution and scoring.

Scoring of hirsutism: Body hair distribution using modified Ferriman-Gallwey score (FGS) [23].

The score used 9 body areas to assess hair growth:

Upper lip, chin, chest, upper back, lower back, upper abdomen, lower abdomen, upper arm and thighs.

Hair growth was rated from 0 (no growth of terminal hair) to 4 (extensive hair growth) in each of the nine locations. A patient score therefore ranged from a minimum score of 0 to a maximum score of 36 .

1 - 8: Constitutional hirsutism.

9 - 30: Symptoms of a disease.

31 - 36: Symptoms of a tumor.

\subsection{Scoring of Acne [24]}

- Mild: Open and closed comedones with few inflammatory papules and pustules.

- Moderate: Papules and pustules, mainly on face.

- Moderately severe: Numerous papules and pustules, and occasional inflamed nodules on face, chest and back.

- Severe: Many large painful nodules, pustules and cysts.

\subsection{Investigations}

Hormonal assay:

Serum FSH \& LH levels, mean LH/FSH ratio were obtained, a ratio $\geq 2.0$ is suggestive of PCOS [25] and Total Testosterone level also was measured.

- Time: on the morning of the $2^{\text {nd }}-5^{\text {th }}$ day of the menstrual cycle after overnight sleep.

- For women with amenorrhea, withdrawal of bleeding occurred by administration of $10 \mathrm{mg}$ medroxyprogesterone acetate twice daily for 5 consecutive days.

- Method of the assay: a venous blood sample $(5 \mathrm{cc})$ was collected under aspetic conditions in a tube and allowed to clot for 30 minutes then centrifuged for 15 minutes to clarify the serum which was stored at $-20^{\circ} \mathrm{C}$ until the analysis.

Ultrasonic examination:

All participants in the study had a 2 dimensions trans-vaginal pelvic or trans-abdominal ultrasound scan. The diagnosis of PCOS by U/S requires the presence of 12 or more follicles per ovary each measuring $2-9 \mathrm{~mm}$ in diameter, and/or increase ovarian volume (>10 ml) [26].

- Trans-vaginal pelvic ultrasound examination was performed with transvaginal probe. 
- The ovaries were examined after the longest medial axis of the ovary had been determined, the second dimension was measured, and then the vaginal probe was rotated 90 degrees to obtain the third dimension.

- Ovarian volume was calculated according to a simplified formula for an ellipsoid $(0.5 \times$ length $\times$ width $\times$ thickness). Ovarian volume per ovary (OVPO) was defined as the average of the ovarian volume obtained from both ovaries.

- Each ovary was scanned in both longitudinal and transverse cross-section from the inner to the outer margins to enumerate the total number of follicles.

- All follicles between 2 and $9 \mathrm{~mm}$ in diameter were counted. The dominant follicle was $(>10 \mathrm{~mm})$.

- The follicle number per ovary (FNPO) was defined as the average for the total number of follicles counted from both ovaries.

\subsection{Data Management and Analysis}

The collected data was revised, coded, tabulated and introduced to a PC using Statistical package for Social Science (SPSS 20). Data was presented and suitable analysis was done according to the type of data obtained for each parameter.

1) Descriptive statistics:

a) Mean, Standard deviation $( \pm S D)$ for numerical data.

b) Frequency and percentage of non-numerical data.

2) Analytical statistics:

a) Student $T$ Test was used to assess the statistical significance of the difference between two study group means.

b) Chi-Square test was used to examine the relationship between two qualitative variables.

c) Fisher's exact test: was used to examine the relationship between two qualitative variables when the expected count is less than 5 in more than $20 \%$ of cells.

d) Correlation analysis (using Spearman method): To assess the strength of association between two quantitative variables. The correlation coefficient denoted symbolically "rs" defines the strength (magnitude) and direction (positive or negative) of the linear relationship between two variables.

- $\mathrm{r}=0-0.19$ is regarded as very weak correlation

- $\mathrm{r}=0.2-0.39$ as weak correlation

- $\mathrm{r}=0.40-0.59$ as moderate correlation

- $\mathrm{r}=0.6-0.79$ as strong correlation

- $\mathrm{r}=0.8-1$ as very strong correlation

\section{Results}

The study included 134 female participants had met the criteria to be diagnosed as PCOS, the range of age was $18-35$ years, with a mean (26.31 \pm 4.47 years). The number of patients with oligomenorrhea was 107 (80.6\%) and with PCOS ultrasound findings were 127 (94.8\%). The range of LH/FSH ratio of the studied 
cases was $0.28-5.68 \mathrm{mIU} / \mathrm{L}$ with a mean $(1.76 \pm 0.94 \mathrm{mIU} / \mathrm{L})$ and range of total testosterone was $0.1-9.5$ with a mean $(1.44 \pm 1.59 \mathrm{ng} / \mathrm{ml})$. Among the study sample $32.1 \%$ of patients had high mean $\mathrm{LH} / \mathrm{FSH}$ ratio (equal or more than 2 ) and $35 \%$ of patients had elevated total testosterone levels.

All participants of the study meeting criteria of PCOS had at least one dermatological findings. Cutaneous disorders that were detected included hirsutism, acne, acanthosis nigricans, androgenic alopecia and seborrheic dermatitis (Table 1). Among the PCOS patients at least one cutaneous disorder was found in 18 patients (13.4\%), two cutaneous disorders in 44 patients $(32.8 \%)$, three cutaneous disorders in 47 patients (35.1\%), four cutaneous disorders in $24(17.9 \%)$ and five cutaneous disorders were found in only one patient $(0.7 \%)$.

1) Hirsutism: Women among study group cases had different severity scores ranging from $8-28$ with mean (17.22 \pm 5.66$)$. Statistically, there was a significant positive correlations between hirsutism and LH/F.S.H ratio( $\mathrm{r}=0.933, \mathrm{p}<$ $0.001)$, hirsutism and total testosterone level $(r=0.209, p=0.019)$ (Figure $1 \&$ Figure 2).

2) Acne was found in $66(49.2 \%)$. Acne grade among PCOS patients were, mild in $19(14.2 \%)$ patients, moderate in $18(13.4 \%)$ patients and severe in 29 (21.6\%).

Statistically, there was a significant positive correlations between acne and LH/F.S.H ratio $(\mathrm{r}=0.91, \mathrm{p}<0.001)$ and total tetesterone level $(\mathrm{r}=0.724, \mathrm{p}<$ 0.001) (Figure 3 and Figure 4).

Correlation between severity of Acne and Hirsutism

Statistically, there was a significant correlation between severity of acne and hirsutism $(r=0.941, \mathrm{p}$ value $<0.001)$ (Figure 5).

3) Acanthosis nigricans

Acanthosis nigricans was present in 72 (53.7\%). Statistically, there was a significant difference between patients with and without acanthosis nigricans regarding LH/F.S.H ratio and total testosterone level ( $\mathrm{p}<0.001)($ Table 2).

Table 1. Dermatological findings among PCO patients.

\begin{tabular}{cccc}
\hline & & N & $\%$ \\
\hline \multirow{2}{*}{ Hirsutism } & Absent & 9 & $6.7 \%$ \\
& Present & 125 & $93.3 \%$ \\
Acne & Absent & 68 & $50.7 \%$ \\
& Present & 66 & $49.2 \%$ \\
Acanthosis nigricans & Absent & 62 & $46.3 \%$ \\
& Present & 72 & $53.7 \%$ \\
Androgenetic alopecia & Absent & 86 & $64.2 \%$ \\
& Present & 48 & $35.8 \%$ \\
Seborrheic dermatitis & Absent & 97 & $72.4 \%$ \\
& Present & 37 & $27.6 \%$ \\
\hline
\end{tabular}




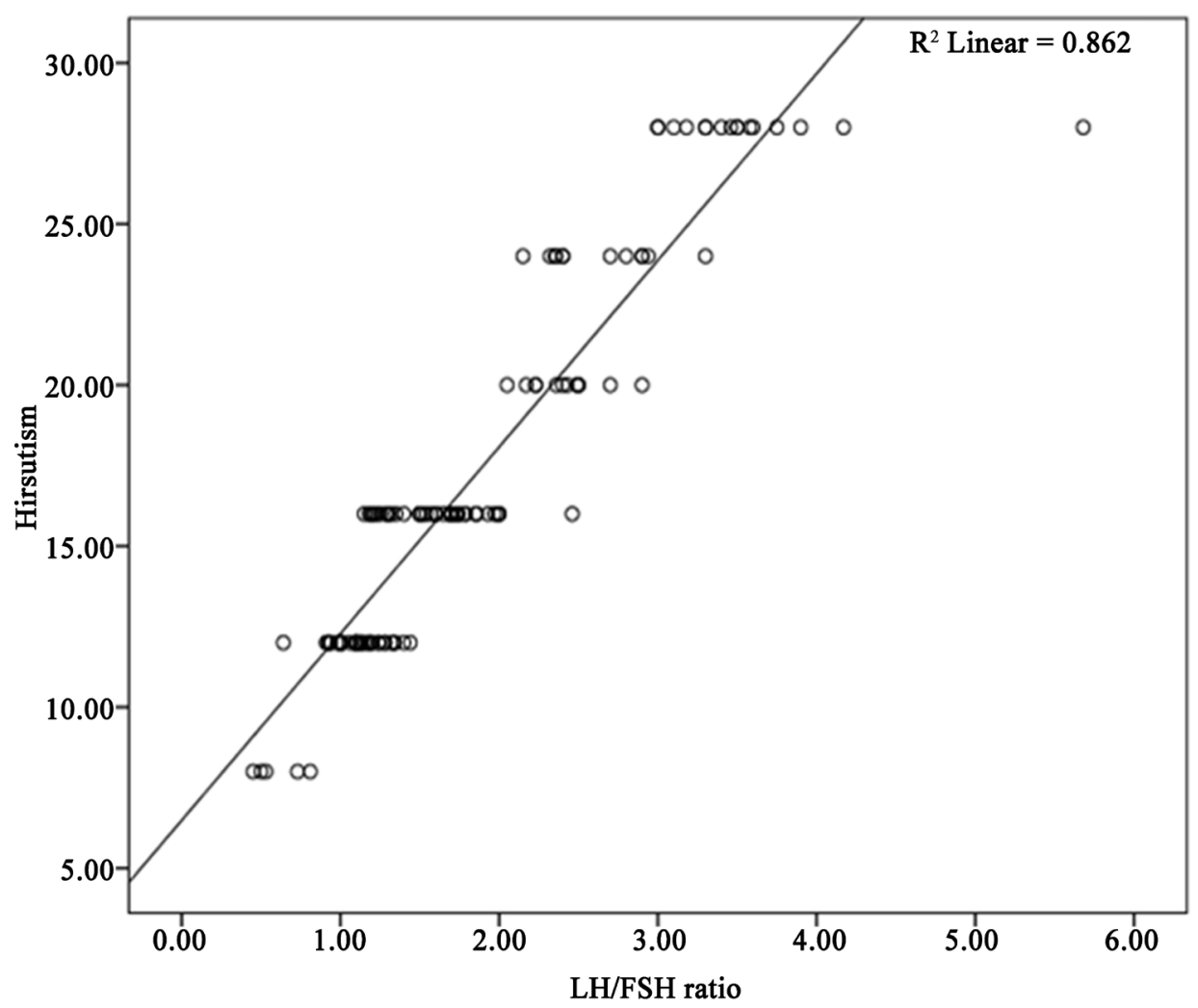

Figure 1. Positive correlation between hirsutism severity and LH/FSH ratio.

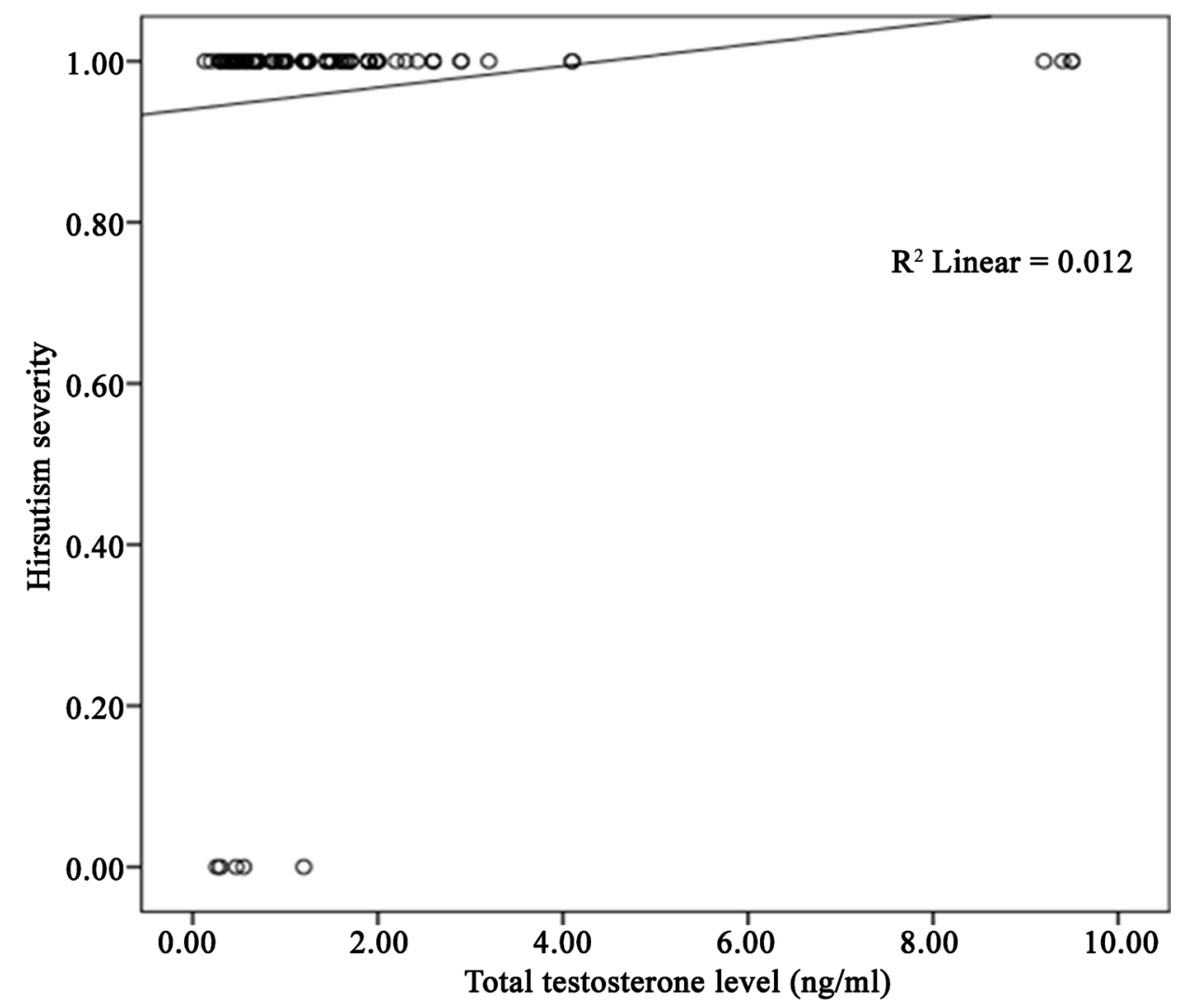

Figure 2. Positive correlation between hirsutism severity and total testosterone levels (ng/ml). 


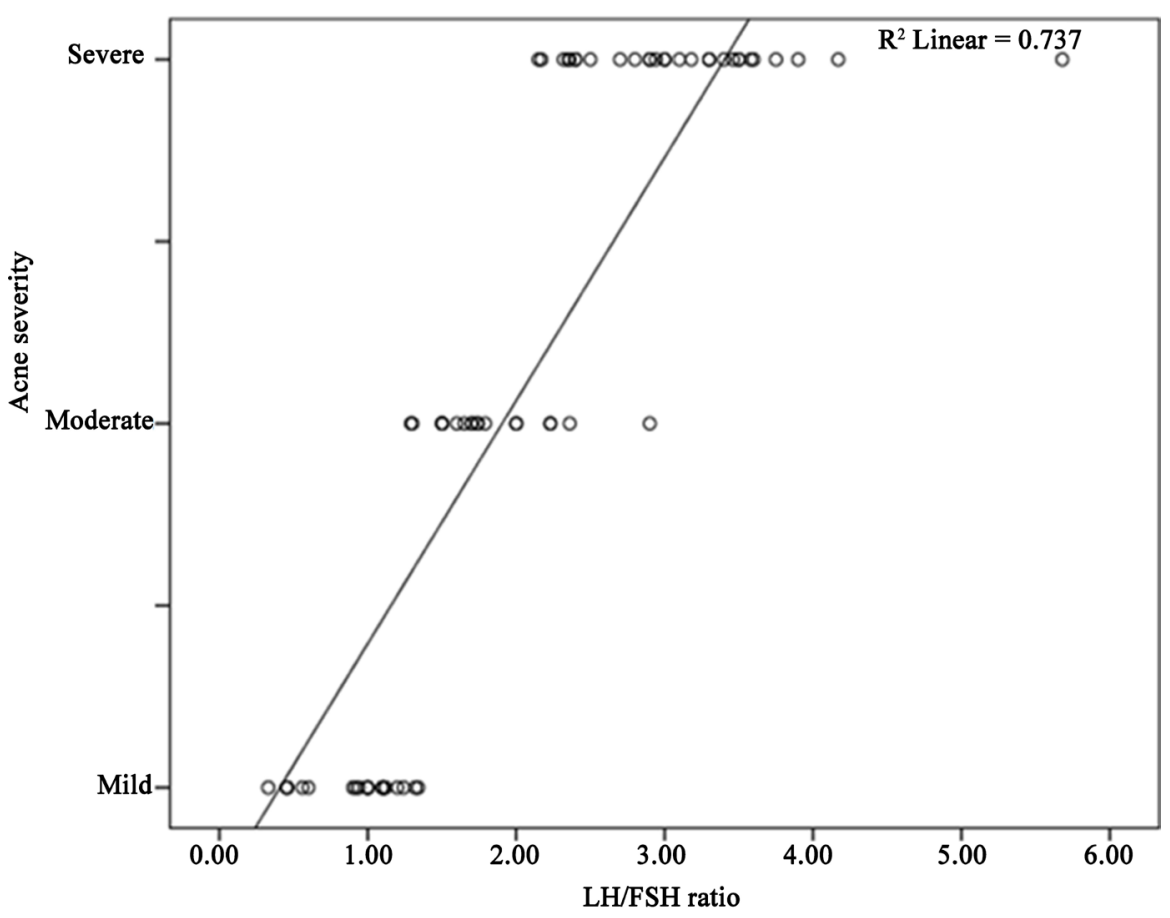

Figure 3. Positive correlation between severity of acne and LH/FSH ratio.

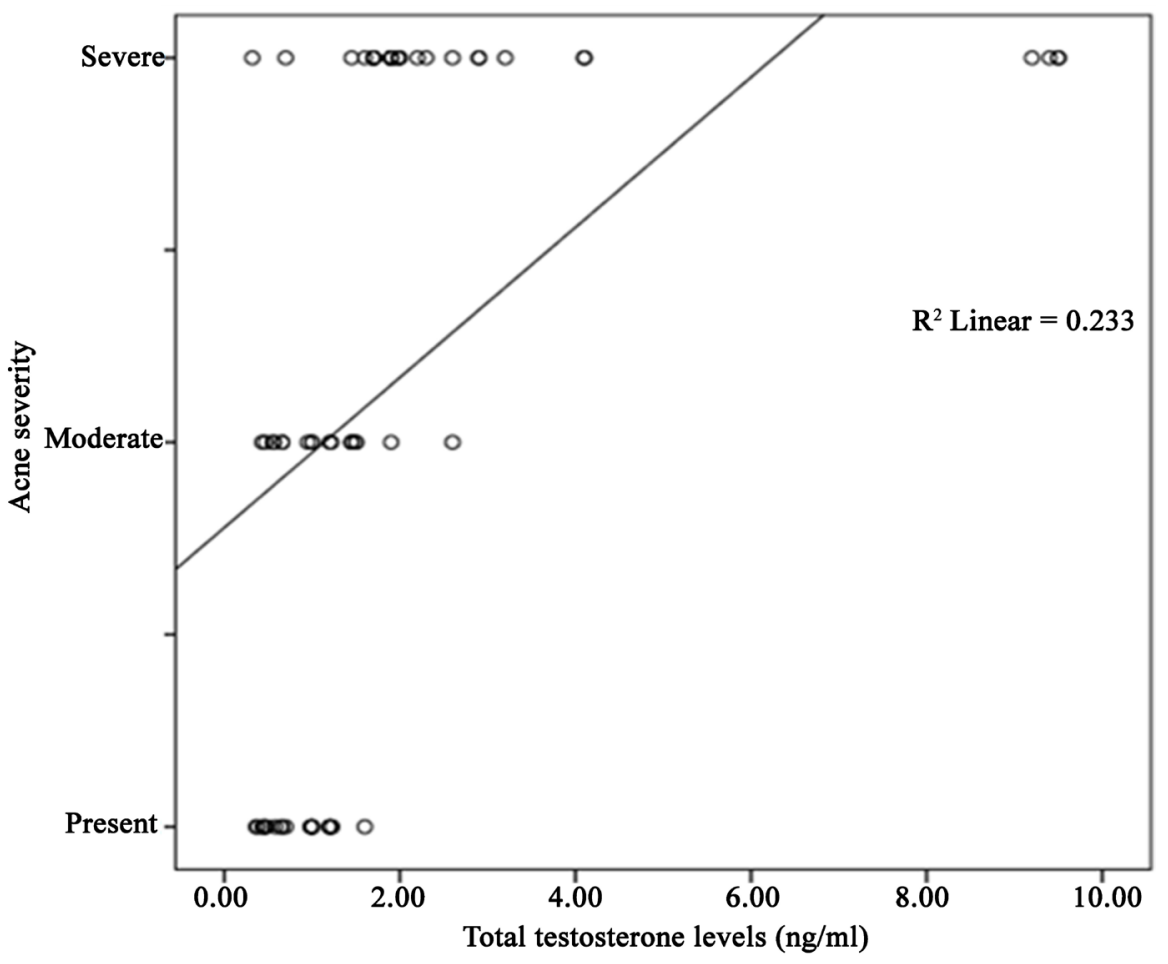

Figure 4. Positive correlation between severity of acne and total testosterone (ng/ml).

4) Androgenetic alopecia:

Androgenetic alopecia was found in 48 (35.8\%). Statistically, there was non-significant difference between patients with and without androgentic alopecia regarding LH/F.S.H ratio and total testosterone level ( $p>0.5)$ (Table 3 ). 
5) Seborrheic dermatitis

Seborrheic dermatitis was found in 37 (27.6\%). Statistically, there was non-significant difference between patients with and without seborrheic dermatitis regarding LH/F.S.H ratio and total testosterone level ( $p>0.5)$ (Table 4).

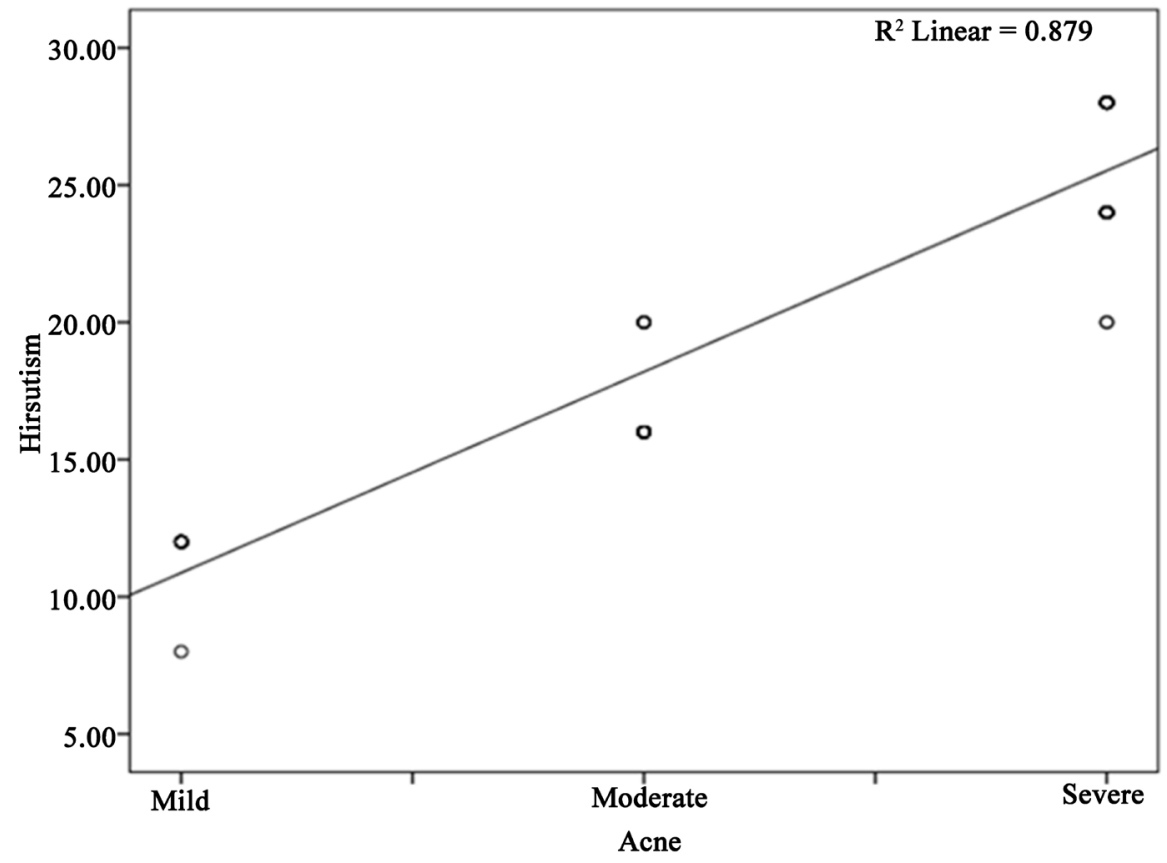

Figure 5. Positive correlation between severity of acne and hirsutism

Table 2. Comparison between patients with and without acanthosis nigricans regarding LH/F.S.H ratio and total testosterone level.

\begin{tabular}{ccccccc}
\hline & \multicolumn{3}{c}{ Acanthosis nigricans } & & t test \\
\cline { 2 - 6 } & \multicolumn{2}{c}{ Absent } & \multicolumn{2}{c}{ Present } & & \\
\cline { 2 - 6 } & Mean & SD & Mean & SD & p value & sig. \\
\hline $\begin{array}{c}\text { LH/FSH ratio } \\
\text { Total testosterone } \\
(\text { ng/ml) }\end{array}$ & 1.0827 & 0.3842 & 2.3449 & 0.8832 & $<0.001$ & $\mathrm{~S}$ \\
\hline
\end{tabular}

${ }^{*}$ Mann Whitney test.

Table 3. Comparison between patients with and without androgenetic alopecia regarding LH/F.S.H ratio and total testosterone level.

\begin{tabular}{ccccccc}
\hline & \multicolumn{3}{c}{ Androgenetic alopecia } & & t test \\
\cline { 2 - 6 } & \multicolumn{2}{c}{ Absent } & \multicolumn{2}{c}{ Present } \\
\cline { 2 - 6 } & Mean & SD & Mean & SD & p value & sig. \\
\hline $\begin{array}{c}\text { LH/FSH ratio } \\
\text { Total testosterone } \\
(\mathrm{ng} / \mathrm{ml})\end{array}$ & 1.7570 & 0.8225 & 1.7679 & 1.1294 & 0.953 & NS \\
& 1.00 & $0.58-1.65$ & 0.86 & $0.45-1.625$ & $0.473^{*}$ & NS \\
\hline
\end{tabular}

${ }^{\star}$ Mann Whitney test. 
Table 4. Correlation between seborrheic dermatitis and other findings.

\begin{tabular}{cccccccc}
\hline & \multicolumn{4}{c}{ Seborrheic dermatitis } & \multicolumn{2}{c}{ t test } \\
\cline { 2 - 6 } & \multicolumn{2}{c}{ Absent } & \multicolumn{2}{c}{ Present } & & \\
\cline { 2 - 6 } & Mean & SD & Mean & SD & p value & sig. \\
\hline BMI & 26.5015 & 3.3287 & 26.2730 & 3.3918 & 0.724 & NS \\
LH/FSH ratio & 1.7452 & 0.9442 & 1.8022 & 0.9397 & 0.755 & NS \\
Total testosterone (ng/ml) & 1.00 & $0.64-1.65$ & 0.86 & $0.43-1.5$ & $0.210^{*}$ & NS \\
\hline
\end{tabular}

${ }^{*}$ Mann Whitney test.

\section{Discussion}

Stein and Leventhal [1] recognized an association between the presence of bilateral polycystic ovaries and signs of amenorrhea, oligomenorrhea, hirsutism and obesity. These manifestations represented the criteria of what is known as Stein Leventhal syndrome [1].

Androgen excess is the main pathology in PCOS. Metabolic abnormalities, insulin resistance, hyperinsulinism, type II diabetes mellitus, endometrial carcinoma, dyslipidemia and psychosocial dysfunction are presented also by PCOS patients [20].

Polycystic ovarian syndrome being one of the most frequent endocrine disorders in the reproductive aged women, is a syndrome of ovarian dysfunction [27]. It is an important cause of both menstrual irregularity and androgen excess in women. When fully expressed, its manifestations include irregular menstrual cycles together with hirsutism and/or acne. Obesity is a frequent concomitant finding [28].

The diagnosis of PCOS is based on a combination of clinical, biological, and ultrasound criteria that have been used variably to define PCOS. Nevertheless, there are no specific or conclusive markers to diagnose this syndrome. Diagnostic criteria and PCOS definitions used by clinicians and researchers are almost as heterogeneous as the syndrome [29].

It was evident that the main pathology in PCOS is androgen excess, however, it was clearly denoted that a proportion of patients with PCOS might not demonstrate overt abnormality in circulating androgens [30]. Excess androgen production by ovary and occasionally by adrenal glands is responsible for the cutaneous changes such as acne, seborrhea, androgenic alopecia and hirsutism [31].

Elevated levels of luteinizing hormone ( $\mathrm{LH}$ ) and subsequent influences on the thecal compartment of the ovary may play an additional role in the establishment of clinically apparent hyper androgenism. Androgen suppression following the diagnosis of hyperandrogenism is the mainstay of treatment in patients with PCOS [32].

It has been reported that women that met the PCOS criteria had a higher prevalence of skin findings. Acne, hirsutism and acanthosis nigricans were the most commonly reported skin disorders [33]. 
The aim of the current study was to define and specify the prevalence of cutaneous disorders of PCOS and indicate the cutaneous lesions that have reliable association with PCOS. These cutaneous disorders can be used as a specific marker to early diagnose or highly suspect patients with PCOS.

The present study included (134) female patients that were diagnosed as having polycystic ovary syndrome according to Rotterdam criteria. All patients were exposed to comprehensive skin examination and transvaginal or abdominal ultrasonography.

Our results showed that the LH: FSH ratio was observed to be more than 2 in $32.1 \%$ of patients, which agree with other data in literature where there was a significant high LH/FSH ratio in 27.5\% PCOS women [34] [35]. Excessive LH secretion relative to FSH by the pituitary gland was the first laboratory abnormality identified in classic PCOS. It is thought to play a role in the pathogenesis of PCOS by increasing androgen production and secretion by ovarian theca cells with subsequent appearance of clinical signs of hyperandrogenism [36].

The total testosterone levels in the current study were observed to be more than $0.68 \mathrm{ng} / \mathrm{ml}$, which indicates biochemical hyperandrogenemia in $35 \%$ of patients. This finding agrees with previous reports who found a significant total testosterone elevation in PCOS women [34] [37]. Liou et al. [38] reported that $46 \%$ of women with PCOS had elevated total testosterone levels, while Ramanand et al. [39] found testosterone levels to be normal or low in all of their patients which might be explained by testing a small sample size ( 50 subjects).

The oligomenorrhea was observed in (81\%) patients which agree with previous reports that indicated oligomenorrhea as the most common presenting symptom in 99.3\% PCOS women [37]. The ultrasound findings were observed in (95\%) of patients which agreed with Pichai et al. [37] who found ultrasonographic features of polycystic ovary in $81.4 \%$ of participants in PCOS women. This denotes that patients of the current study fulfill the required diagnostic criteria of Rotterdam which are essential for the diagnosis of PCOS.

The current study showed the presence of at least one skin finding in every patient. There was also a very high prevalence of hirsutism among PCOS patients $(93 \% ; 125 / 134)$. Such finding is in good agreement with previous studies that have reported high prevalence of hirsutism in PCOS women estimating 28\% [38], 62.5\% [35] and 58.1\% [40], which further supports that hirsutism is the most common skin manifestation in PCOS [33]. The higher prevalence found in our finding might be attributed to the fact that all our patients were evaluated after properly diagnosed to have PCOS. Thus, hirsutism might be considered as a cutaneous marker in PCOS patients.

Examination and scoring of hirsutism revealed score ranging from 8 - 28 denoting a profound hyperandrogenic state in our PCOS patients, that was demonstrated in positive correlation between hirsutism versus both LH/F.S.H ratio and total testosterone level $(\mathrm{p}<0.05)$. These findings are consistent with those of Vural et al. [32], Mardanian and Heidari [41] that indicated a positive correla- 
tion of high hirsute Ferriman Gallwey scores with LH/ FSH ratio and total testosterone serum levels.

Our finding of positive correlation between hirsutism and hormonal abnormalities (increased LH/FSH ratio and total testosterone) upholds and further indicates that hirsutism is an important indication for a reproductive and metabolic workup in suspected cases of PCOS.

The current study showed prevelance of acne among PCOS patients (49.2\%; $66 / 134)$. Such finding is in good agreement with previous studies that have reported high prevelance of acne in PCOS women estimating 48\% [38], 67.5\% [35], 95\% [42], 56.6\% [37] and 61.8\% [40].

Examination and scoring of acne revealed a high severity score denoting a profound hyperandrogenic state in our PCOS patients. This was demonstrated also in the positive correlation between acne versus both LH/F.S.H ratio and total testosterone level $(\mathrm{p}<0.05)$. These findings are consistent with those of Jayaram et al. [43] who reported a positive correlation between acne versus both high LH and total testosterone levels. On the contrary Hong et al. [42] noticed that acne grade and number of acne lesions were not significantly different between the hyperandrogenic and nonhyperandrogenic group. This clarified that although androgen is definitely a critical hormone that elicits and aggravates acne through excessive sebum production, yet the serum androgen levels might not be directly proportional to local androgen levels near the pilosebaceous unit, perhaps explaining why most patients with acne have normal androgen levels. Acne commonly results from the local effects of increased androgen receptor sensitivity within the pilosebaceous unit.

Schmidt et al. [2] however observed that acne was a common manifestation of PCOS patients but it was not reliable for PCOS because among women with PCOS, acne when present was not associated with metabolic dysregulation or increased serum androgens and suggesting that the acne-androgen association is a complex one.

Our finding of positive correlation between acne and hormonal abnormalities (increased LH/FSH ratio and total testosterone) might indicate that acne is a potential indication for a reproductive and metabolic workup in suspected cases of PCOS. Thus, acne might be considered as a cutaneous marker in PCOS patients.

The current study showed prevalence of acanthosis nigricans in $53.7 \%$; 72/134) of PCOS patients, such finding is in good agreement with previous studies that have reported high prevalence of acanthosis nigricans in PCOS women estimating $44.16 \%$ [39], 22.5\% [35] and $15.8 \%$ [40]. The high prevelence in our finding might be attributed to the fact that all our patients were evaluated after properly diagnosed to have PCOS. Thus, AN might be considered as a cutaneous marker in PCOS patients. Cruz et al. [44] explained that the proliferation of keratinocytes is caused by hyperinsulinaemia and increased binding of excessive serum insulin to IGF-1 receptors in the peripheral tissues because insulin resistance is often observed in PCOS, So acanthosis nigricans might be a common 
cutaneous manifestation in PCOS.

Our results showed that there was a significant difference between patients with and without acanthosis nigricans regarding $\mathrm{LH} / \mathrm{FSH}$ ratio and total testosterone level ( $\mathrm{p}<0.001$ ) which may be explained by the association of AN with hyperinsulinemia, that can promote ovarian thecal androgen secretion and inhibiting hepatic synthesis of sex hormone-binding globulin [45].

The current study showed prevalence of androgenic alopecia in $(35.8 \%$; 48/134) of PCOS patients, such finding is in good agreement with previous studies that have reported comparable percentage of prevalence of androgenic alopecia in PCOS women (30\%) Gowri et al. [35] and (23\%) Feng et al. [40]. Hong et al. [42] have shown that the proportion of PCOS patients who had androgenetic alopecia was $12.5 \%$ which was slightly higher than that reported from western populations. This discrepancy can be driven from the ethnic differences and the difficulty of a definitive diagnosis.

Our results also showed that there was no significant difference between patients with and without androgenetic alopecia regarding LH/F.S.H ratio and total testosterone level ( $\mathrm{p}>0.5$ ). Schmidt et al. [2] and Feng et al. [40] have reported no statistically significant relationship between hyperandrogenism and central hair loss pattern. In contrast, [35] reported that androgenetic alopecia was associated with an elevated $\mathrm{LH}$ level in $15 \%$ of patients. This difference might be attributed to the difference in sample size (40 patients versus 134 in our study). Our data support previous observations that AGA in PCOS is more tightly associated with clinical hyperandrogenism but not biochemical hyperandrogenism. This may be due to the ethnic differences in the number of hair follicles present and individual skin sensitivity of the pilosebaceous unit to androgens.

According to Schmidt and Shinkai [33]: acne, hirsutism, and AN were the most common skin manifestations, while hirsutism and AN were the most informative for PCOS diagnosis. The current study showed that hirsutism, acne and AN are also the most common and reliable skin manifestations for PCOS. These cutaneous manifestations commonly associated with PCOS due to the positive correlation found between these cutaneous disorders and the hormonal abnormalities (increased LH/FSH ratio and total testosterone) usually existing in PCOS.

Seborrheic dermatitis was seen in $27.6 \%$ of patients. Schmidt and Shinkai [33] observed seborrheic dermatitis in $30.4 \%$ of patients and Feng et al. [40] found it in $38.2 \%$ of their patients. Our results showed that, there was a non-significant difference between patients with and without seborrheic dermatitis regarding LH/F.S.H ratio and total testosterone level ( $p>0.5$ ).

Seborrheic dermatitis in our PCOS patients was contradictory with other studies. Since our study had a small number of patients, this may be the reason for the inconsistent findings observed.

\section{Conclusion}

Findings in our study showed the possible association of hirsutism, acne, AN, 
AGA and seborrheic dermatitis with PCOS. These cutaneous manifestations could be relied upon as potential cutaneous indicator of PCOS. Using Rotterdam criteria women who met the criteria of PCOS had a higher prevalence of hirsutism, acne, AN, androgenic alopecia and seborrheic dermatitis. Our study demonstrated that the prevalence of cutaneous manifestations in PCOS shows a clinical spectrum ranging from one to multiple findings. Among the PCOS patients at least one cutaneous disorder was found in 18 patients (13.4\%), two cutaneous disorders in 44 patients $(32.8 \%)$, three cutaneous disorders in 47 patients $(35.1 \%)$, four cutaneous disorders in 24 (17.9\%) and five cutaneous disorders were found in only one patient $(0.7 \%)$.

\section{Acknowledgements}

Authors are grateful for the women agreed and gave consent to be included in this study.

\section{Financial Support}

This study was not financially supported by any institutions or organizations.

\section{Ethical Approval}

All procedures performed in studies involving human participants were in accordance with the ethical standards of the institutional and/or national research committee and with the 1964 Helsinki declaration and its later amendments or comparable ethical standards. This study received approval by local institutional ethical committee of Ain Shams University Hospital and informed consent was obtained from all individual participants included in the study.

\section{Availability of Data and Material}

All data are available transparently.

\section{Authors' Contributions}

Mohammed Hussain Mostafa: The corresponding author and responsible for the idea of the study, data collection and analysis, intellectual content, writing and editing the manuscript and final revision before publication.

Nader Fouad Ragab, Ghada Fathy Mohammed: Dermatologic follow up the cases, data collection and revising the manuscript.

\section{Consent to Participate}

Written consent has been acquired from participants after explaining the study design.

\section{Consent for Publication}

All authors have revised and agreed to publication of the study. 


\section{Conflicts of Interest}

The authors declare no conflicts of interest regarding the publication of this paper.

\section{References}

[1] Stein, I. and Leventhal, M. (1935) Amenorrhea Associated with Bilateral Polycystic Ovaries. American Journal of Obstetrics and Gynecology, 29, 181-185. https://doi.org/10.1016/S0002-9378(15)30642-6

[2] Schmidt, T.H., Khanijow, K., Cedars, M.I., Huddleston, H., Pasch, L., Wang, E.T., Lee, J., Zane, L.T. and Shinkai, K. (2016) Cutaneous Findings and Systemic Associations in Women with Polycystic Ovary Syndrome. JAMA Dermatology, 152, 391-398. https://doi.org/10.1001/jamadermatol.2015.4498

[3] Norman, R.J., Dewailly, D., Legro, R.S. and Hickey, T.E. (2007) Polycystic Ovary Syndrome. The Lancet, 370, 685-697. https://doi.org/10.1016/S0140-6736(07)61345-2

[4] Rotterdam ESHRE/ASRM-Sponsored PCOS Consensus Workshop Group (2004) Revised 2003 Consensus on Diagnostic Criteria and Long-Term Health Risks Related to Polycystic Ovary Syndrome (PCOS). Human Reproduction, 19, 41-47. https://doi.org/10.1093/humrep/deh098

[5] Carmina, E., Legro, R.S., Stamets, K., Lowell, J. and Lobo, R. (2003) Difference in Body Weight between American and Italian Women with Polycystic Ovary Syndrome: Influence of the Diet. Human Reproduction, 18, 2289-2293.

https://doi.org/10.1093/humrep/deg440

[6] Lee, A.T. and Zane, L.T. (2007) Dermatologic Manifestations of Polycystic Ovary Syndrome. American Journal of Clinical Dermatology, 8, 201-219. https://doi.org/10.2165/00128071-200708040-00003

[7] Rosenfield, R.L. (2005) Clinical Practice. Hirsutism-New England Journal of Medicine, 353, 2578-2588. https://doi.org/10.1056/NEJMcp033496

[8] Azziz, R. (2006) Diagnosis of Polycystic Ovarian Syndrome: The Rotterdam Criteria Are Premature. Journal of Clinical Endo-Crinology \& Metabolism, 91, 781-785. https://doi.org/10.1210/jc.2005-2153

[9] Marla, E.L., Donna, R.C. and Roger, A. (2008) Pierson Diagnostic Criteria for Polycystic Ovary Syndrome: Pitfalls and Controversies. Journal of Obstetrics and $G y-$ naecology Canada, 30, 671-679. https://doi.org/10.1016/S1701-2163(16)32915-2

[10] Hatch, R., Rosenfield, R.L., Kim, M.H. and Tredway, D. (1981) Hirsutism: Implications, Etiology, and Management. American Journal of Obstetrics and Gynecology, 140, 815-830. https://doi.org/10.1016/0002-9378(81)90746-8

[11] Borgia, F., Cannavò, S., Guarneri, F., Cannavò, S.P., Vaccaro, M. and Guarneri, B. (2004) Correlation between Endocrinological Parameters and Acne Severity in Adult Women. Acta Dermato- Venereologica, 84, 201-204. https://doi.org/10.1080/00015550410023248

[12] Strauss, J.S., Krowchuk, D.P., Leyden, J.J., et al. (2007) Guidelines of Care for Acne Vulgaris Management. Journal of the American Academy of Dermatology, 56, 651-663. https://doi.org/10.1016/j.jaad.2006.08.048

[13] Lucky, A.W., McGuire, J., Rosenfield, R.L., Lucky, P.A. and Rich, B.H. (1983) Plasma Androgens in Women with Acne Vulgaris. Journal of Investigative Dermatology, 81, 70-74. https://doi.org/10.1111/1523-1747.ep12539043 
[14] Barth, J.H., Yasmin, E. and Balen, A.H. (2007) The Diagnosis of Polycystic Ovary Syndrome: The Criteria Are Insufficiently Robust for Clinical Research. Clinical Endocrinology, 67, 811-815. https://doi.org/10.1111/j.1365-2265.2007.02932.x

[15] Goodman, N.F., Bledsoe, M.B., Cobin, R.H., et al. (2001) American Association of Clinical Endocrinologists Medical Guidelines for the Clinical Practice for the Diagnosis and Treatment of Hyperandrogenic Disorders. Endocrine Practice, 7, 120-134. https://doi.org/10.4158/EP.7.2.120

[16] Cela, E., Robertson, C., Rush, K., Kousta, E., White, D.M., Wilson, H., Lyons, G., Kingsley, P., McCarthy, M.I. and Franks, S. (2003) Prevalence of Polycystic Ovaries in Women with Androgenic Alopecia. European Journal of Endocrinology, 149, 439-442. https://doi.org/10.1530/eje.0.1490439

[17] Fraser, I.S. and Kovacs, G. (2004) Current Recommendations for the Diagnostic Evaluation and Follow-Up of Patients Presenting with Symptomatic Polycystic Ovary Syndrome. Best Practice \& Research Clinical Obstetrics \& Gynaecology, 18, 813-823. https://doi.org/10.1016/S1521-6934(04)00106-3

[18] Moura, H.H.G., Costa, D.L.M., Bagatin, E., Sodre, C.T. and Azulay, M.M. (2011) Polycystic Ovary Syndrome: A Dermatologic Approach. Anais Brasileiros de Dermatologia, 86, 111-119. https://doi.org/10.1590/S0365-05962011000100015

[19] Robert, L.B. and David, A.E. (2016) Clinical Manifestations of Polycystic Ovary Syndrome in Adults.

https://www.uptodate.com/contents/diagnosis-of-polycystic-ovary-syndrome-in-ad ults

[20] Azziz, R., Carmina, E., Dewailly, D., et al. (2009) The Androgen Excess and PCOS Society Criteria for the Polycystic Ovary Syndrome: The Complete Task Force Report. Fertility and Sterility, 91, 456. https://doi.org/10.1016/j.fertnstert.2008.06.035

[21] Chae, S.J., Kim, J.J., Choi, Y.M., et al. (2008) Clinical and Biochemical Characteristics of Polycystic Ovary Syndrome in Korean Women. Human Reproduction, 23, 1924-1931. https://doi.org/10.1093/humrep/den239

[22] Teede, H., Deeks, A. and Moran, L. (2010) Polycystic Ovary Syndrome: A Complex Condition with Psychological, Reproductive and Metabolic Manifestations That Impacts on Health across the Lifespan. BMC Medicine, 8, 41.

https://doi.org/10.1186/1741-7015-8-41

[23] Ferriman, D. and Galllwey, J.D. (1961) Clinical Assessment of Body Hair Growth in Women. The Journal of Clinical Endocrinology and Metabolism, 21, 1440-1447. https://doi.org/10.1210/jcem-21-11-1440

[24] Haider, A. and Shaw, J.C. (2004) Treatment of Acne Vulgaris. JAMA, 292, 726-735. https://doi.org/10.1001/jama.292.6.726

[25] Michael, T.S. (2004) Polycystic Ovarian Syndrome: Diagnosis and Management. Clinical Medicine \& Research, 2, 13-27. https://doi.org/10.3121/cmr.2.1.13

[26] Balen, A.H., Laven, J.S., Tan, S.L. and Dewailly, D. (2003) Ultrasound Assessment of the Polycystic Ovary: International Consensus Definitions. Human Reproduction Update, 9, 505-514. https://doi.org/10.1093/humupd/dmg044

[27] Azziz, R., Woods, K.S., Reyna, R., Key, T.J., Knochenhauer, E.S. and Yildiz, B.O. (2004) The Prevalence and Features of the Polycystic Ovary Syndrome in an Unselected Population. The Journal of Clinical Endocrinology and Metabolism, 89, 2745-2749. https://doi.org/10.1210/jc.2003-032046

[28] Ehrmann, D.A. (2005) Medical Progress: Polycystic Ovary Syndrome. The New England Journal of Medicine, 352, 1223-1236.

https://doi.org/10.1056/NEJMra041536 
[29] Kubota, T. (2013) Update in Polycystic Ovary Syndrome: New Criteria of Diagnosis and Treatment in Japan. Reproductive Medicine and Biology, 12, 71-77. https://doi.org/10.1007/s12522-013-0145-1

[30] Escobar-Morreale, H.F., Roldan, B., Barrio, R., Alonso, M., Sancho, J., de la, C.H., et al. (2000) High Prevalence of the Polycystic Ovary Syndrome and Hirsutism in Women with Type 1 Diabetes Mellitus. The Journal of Clinical Endocrinology and Metabolism, 85, 4182-4187. https://doi.org/10.1210/jc.85.11.4182

[31] William, N. and Burns, W.N. (2015) Patient Information: Polycystic Ovary Syndrome-Disease Process and Treatment. http://marshallhealth.org/media/19971/PolycysticOvarySyndrome.pdf

[32] Vural, B., Ozkan, S. and Bodur, H. (2007) Is Prostate-Specific Antigen a Potential New Marker of Androgen Excess in Polycystic Ovary Syndrome? Journal of Obstetrics and Gynaecology Research, 33, 166-173. https://doi.org/10.1111/j.1447-0756.2007.00507.x

[33] Schmidt, T.H. and Shinkai, K. (2015) Evidence-Based Approach to Cutaneous Hyperandrogenism in Women. Journal of the American Academy of Dermatology, 73, 672-690. https://doi.org/10.1016/j.jaad.2015.05.026

[34] Fakhoury, H., Tamim, H., Ferwana, M., Siddiqui, I.A., Adham, M. and Tamimi, W. (2012) Age and BMI Adjusted Comparison of Reproductive Hormones in PCOS. Journal of Family Medicine and Primary Care, 1, 132. https://doi.org/10.4103/2249-4863.104984

[35] Gowri, B.V., Chandravathi, P.L., Sindhu, P.S. and Naidu, K.S. (2015) Correlation of Skin Changes with Hormonal Changes in Polycystic Ovarian Syndrome: A Cross-Sectional Clinical Study. Indian Journal of Dermatology, 60, 419. https://doi.org/10.4103/0019-5154.160505

[36] Rosenfield, L. (2008) What Every Physician Should Know about Polycystic Ovary Syndrome. Dermatologic Therapy, 21, 354-361. https://doi.org/10.1111/j.1529-8019.2008.00217.x

[37] Pichai, L., Thanyarat, W., Suchada, I., Kitirat, T., Manee, R. and Surasak, A. (2016) Correlation of Clinical and Biochemical Hyperandrogenism in Thai Women with Polycystic Ovary Syndrome Japan Society 2 of Obstetrics and Gynecology. Journal of Obstetrics and Gynaecology Research, 42, 678-683. https://doi.org/10.1111/jog.12945

[38] Liou, T.H., Yang, J.H., Hsieh, C.H., Lee, C.Y., Hsu, C.S. and Hsu, M.I. (2009) Clinical and Biochemical Presentations of Polycystic Ovary Syndrome among Obese and Nonobese Women. Fertility and Sterility, 92, 1960-1965. https://doi.org/10.1016/j.fertnstert.2008.09.003

[39] Ramanand, S.J., Ghongane, B.B., Ramanand, J.B., Patwardhan, M.H., Ghanghas, R.R. and Jain, S.S. (2013) Clinical Characteristics of Polycystic Ovary Syndrome in Indian Women. Indian Journal of Endocrinology and Metabolism, 17, 138. https://doi.org/10.4103/2230-8210.107858

[40] Feng, J.G., Guo, Y., Ma, L.A., Xing, J., Sun, R.F. and Zhu, W. (2017) Prevalence of Dermatologic Manifestations and Metabolic Biomarkers in Women with Polycystic Ovary Syndrome in North China. Journal of Cosmetic Dermatology, 17, 511-517. https://doi.org/10.1111/jocd.12387

[41] Mardanian, F. and Heidari, N. (2011) Diagnostic Value of Prostate-Specific Antigen in Women with Polycystic Ovary Syndrome. Journal of Research in Medical Sciences, 16, 999-1005.

[42] Hong, J.S., Kwon, H.H., Park, S.Y., Jung, J.Y., Yoon, J.Y., Min, S., Choi, Y.M. and 
Suh, D.H. (2015) Cutaneous Manifestations of the Subtypes of Polycystic Ovary Syndrome in Korean Patients. European Academy of Dermatology and Venereology, 29, 42-47. https://doi.org/10.1111/jdv.12432

[43] Jayaram, D., Handattu, S., Shetty, P.K. and Banavasi, G.S. (2016) Cutaneous Manifestations in Polycystic Ovary Syndrome: With a Correlation to Selected Hormonal Levels. Indian Journal of Applied Research, 6, 11-22.

[44] Cruz, P.D. and Hud, J.A. (1992) Excess Insulin Binding to Insulin-Like Growth Factor Receptors: Proposed Mechanism for Acanthosis Nigricans. Journal of Investigative Dermatology, 98, 82S-85S. https://doi.org/10.1111/1523-1747.ep12462293

[45] Poretsky, L., Cataldo, N.A., Rosenwaks, Z. and Giudice, L.C. (1999) The Insulin-Related Ovarian Regulatory System in Health and Disease. Endocrine Reviews, 20, 535-582. https://doi.org/10.1210/edrv.20.4.0374 